\title{
Social Shopping: The Good The Bad and The Ugly
}

\author{
Gabrielle Peko \\ University of Auckland \\ g.peko@auckland.ac.nz
}

\author{
Valeria Sadovykh \\ University of Auckland \\ ahlerka@hotmail.com
}

\author{
David Sundaram \\ University of Auckland \\ d.sundaram@auckland.ac.nz
}

With the emergence in online social networks (OSN) like Facebook and Twitter, more studies appear with regard to information search using OSN. Online social media such as blogs, wikis, and social networks are improving speed and reinventing communication. Furthermore, the burgeoning use of OSN is changing our e-commerce society from transaction-based to relationship-based. OSN are increasingly being used to obtain information, opinions, recommendations, and comparisons and to view discussions to make shopping decisions. Often consumers are faced with purchase dilemmas and there are many questions in one's mind that could potentially affect the outcome of the purchase decision.

How shopping decisions are taken with the support of OSN and how these networks influence purchase behavior has not been explored sufficiently in research. Although the usage of OSN is growing rapidly, there is a poor understanding of how OSN can provide support and influence purchase decisions in general.

The objective of this mini-track is to obtain insights and develop theoretical and practical understanding on topics and issues related to the influence of OSN on consumption orientated shopping decisions.

The mini-track welcomed conceptual, theoretical, and empirical papers that enrich our understanding of OSN and its design and how they support, influence and manipulate shopping decisions. All methodological approaches were appreciated.

Topics of interest included but were not limited to:

- Shopping Decision Making and Decision Support

- Co-creation of value on multisided platforms

- Group shopping sites, communities, and marketplaces

- Influence, persuasion, and peer pressure

- Market manipulation and incentives

- Advertising, marketing and recommender systems

- Commerce, mobile commerce, and social commerce

- Shopping gamification and shopping games

- Consumerism, compulsive, and addictive shopping

- Fraud, deception, governance, risk, compliance, security, and privacy

- Gen-X, Y, Z, millennial shopping

- Age, gender, and demographics

- OSN post purchase cognitive dissonance

- Processes, systems, tools and technologies to support social shopping

The mini track continues to attract a variety of interesting papers. This year we had several excellent papers. Of these papers, two in particular investigated key issues that exist in the research area. The first paper proposes a methodology that 
supports the data consumer to reduce their risk in investing in a data set. The aim of this approach is to enable a fair data trading process that considers the requirements of the data provider and the data consumer. The 21 st century is the century of data. Data are the new oil and the fuel of most new applications and business models. The increasing value of data leads to the fact, that possibilities and methods become necessary to trade with this commodity "data". One of these possibilities is a data marketplace, a digital ecommerce platform like eBay or Amazon, dedicated to trading data, information, and data sets. Since data and information are not so easy to verify in contrast to physical goods, methods are required to characterize them but also to check them against user requirements. Here, we have a conflict between the security and privacy interests from a data provider on the one side and the necessity to check the consumer requirements on the other side. In this paper, the specific challenges that arise in data trading are discussed and a methodology on how a user can check his specific requirements (Data Quality) is introduced.

The second paper explores how research conducted on Dark Web cryptomarkets and drug trading imageboards could protect data and the communities being investigated while carrying out needed research. In recent years, there have increasingly been conflicting calls for more government surveillance online and, paradoxically, increased protection of the privacy and anonymity of individuals. Many corporations and groups globally have come under fire for sharing data with law enforcement agencies as well as for refusing to cooperate with said agencies, in order to protect their customers. The focus of the research study is Dark Web drug trading sites as an exemplary case of problematic areas of information protection, and what practices should be followed when gathering data from the Dark Web. Using lessons from an ongoing research project, best practices are outlined for protecting the safety of the people under study on these sites without compromising the quality of research data gathering. 